\title{
H19 contributes to poor clinical features in NSCLC patients and leads to enhanced invasion in A549 cells through regulating miRNA-203-mediated epithelial-mesenchymal transition
}

\author{
XIAO-JUN GE, LI-MEI ZHENG, ZHONG-XIN FENG, MEI-YONG LI, LAN LIU, YU-JIE ZHAO and JUN-YAO JIANG
}

Department of Laboratory Medicine, Affiliated Hospital of Zunyi Medical College, Zunyi, Guizhou 563003, P.R. China

Received January 31, 2018; Accepted June 28, 2018

DOI: $10.3892 / \mathrm{ol} .2018 .9187$

\begin{abstract}
Recent studies have demonstrated that the overexpression of H19 may contribute towards development of tumorigenesis in various types of cancer. To investigate the role of $\mathrm{H} 19$ in the development of non-small cell lung cancer (NSCLC), 76 NSCLC tissues samples and their adjacent normal tissue samples were collected. Expression level of $\mathrm{H} 19$, and its association with clinicopathological features and overall survival was analyzed. It was found that compared with normal adjacent tissues, H19 expression was elevated in NSCLC tissues along with a decreased miR-203 expression level. It was also found that patients who were in advanced clinical stages had a higher H19 and a lower miR-203 expression compared to normal tissues. The overall survival time of patients with higher H19 expression was shorter compared with the lower H19 expression group. Upregulation of A549 enhanced cell proliferation and promoted invasion. Overexpression of H19 stimulated the epithelial-mesenchymal transition (EMT) process in lung cancer cells and demonstrated typical morphological characteristics of EMT. The level of mesenchymal marker protein, such as Vimentin and SNAI1 increased; while CDH1 protein level decreased. Also, H19 negatively regulated miR-203. Inhibition of H19 attenuated miR-203 induced EMT process. Upregulation of $\mathrm{H} 19$ contributes to poor clinical features in patients with NSCLC, induces occurrence of EMT, promotes proliferation and stimulates cell invasion in NSCLC cell line through regulating miRNA-203 mediated EMT.
\end{abstract}

Correspondence to: Professor Xiao-Jun Ge, Department of Laboratory Medicine, Affiliated Hospital of Zunyi Medical College, 138 Dalian Road, Zun Yi, Guizhou 563003, P.R. China E-mail: gxj_199421@163.com

Key words: microRNA, non-small cell lung cancer, epithelialmesenchymal transition, long non-coding RNA

\section{Introduction}

Lung cancer is a highly malignant human cancer in the world as its mortality rate is highest in contrast to other types of cancers (1). In addition, non-small cell lung cancer (NSCLC) accounts for 75 to $80 \%$ pathological types in all lung cancers (2). Although there has been much progress in the study of NSCLC and its treatment, poor prognosis associated with lung cancer remains a serious problem for patients (3). Therefore, effective early diagnosis and prognostic markers can be significant and can enhance clinical value for NSCLC. The discovery of new anti-tumor molecules, as potential targets for clinical prevention and treatment of lung cancer, will create a new approach to tackle lung cancer treatment.

Long non-coding RNA (lncRNA) is a group of RNA with $>200 \mathrm{nt}$ length and is located in the nucleus or cytoplasm (4). Although lncRNA does not have the function of an encoded protein, its finding promotes the progress of research in the field of non-coding RNA (5). Recent studies found that lncRNA participates in the process of $\mathrm{X}$ chromosome gene silencing, genomic imprinting, chromatin modification, transcriptional activation and regulation of gene expression in the nucleus and other transcription processes (6-8). Studies found that lncRNA is involved in many biological processes such as apoptosis and cell cycle (9). Also, IncRNA plays a crucial role in human disease occurrence and development processes $(10,11)$. Therefore, the role of lncRNA in tumors draws considerable attention.

H19 was one of the earliest lncRNA groups identified by researchers. Naturally, it is a highly conserved lncRNA that is found in mammals and it is approximately $2.3 \mathrm{~kb}$ in length (12). Previous studies confirmed that H19 plays an important role in embryonic development, and it is abnormally expressed in a variety of tumors including bladder cancer, gastric cancer and hepatocellular carcinoma through regulating proliferation, invasion and metastasis of tumors (13-15). However, the role of H19 in NSCLC and its underlying mechanisms are unclear. Our study compared the expression of H19 in NSCLC tissues and adjacent tissues. Also, the association between differential expression and clinicopathological parameters of NSCLC was also analyzed. Furthermore, in vitro experiments investigated the influence of H19 on NSCLC cell proliferation, 
epithelial-mesenchymal transition (EMT) impact and invasion ability. Also, the potential regulation mechanisms of $\mathrm{H} 19$ induced EMT process was investigated.

\section{Materials and methods}

Clinical features. A total of 76 NSCLC tissue samples were collected from patients (age 45-78 years; 32 males and 44 females) who underwent thoracic surgery from 2009 to 2014 in The First Affiliated Hospital of Zunyi Medical University. Adjacent non-tumor tissues were obtained at least $2 \mathrm{~cm}$ away from the tumor edges (no observed cancer cells under endoscopic) and used as controls. The clinical stage and histological classification was evaluated with the National Comprehensive Cancer Network (NCCN) NSCLC Guidelines (v.7. 2015). Resection specimens were stored into liquid nitrogen. The study was approved by the Ethics Committee of Zunyi Medical University. Informed consent was obtained from each patient prior to surgery. All experimental procedures were carried out in accordance with the approved guidelines and were in agreement with the Declaration of Helsinki.

Cell culture. Normal bronchial epithelial cell line BEAS-2B, NSCLC adenocarcinoma cell line A549, SPC-A1 and squamous cell SKMES-1 were purchased from the Shanghai Institute of Life Sciences Institute of Biochemistry and the Cell Biology Institute of Cell Bank (Shanghai, China). All cell lines were cultured in RPMI-1640 containing $10 \%$ fetal bovine serum (Sigma-Aldrich; Merck KGaA, Darmstadt, Germany), $100 \mathrm{U} / \mathrm{ml}$ penicillin and $100 \mathrm{mg} / \mathrm{ml}$ streptomycin and were placed in $37^{\circ} \mathrm{C}$ cell incubation supplied with $5 \% \mathrm{CO}_{2}$. Culture medium was replaced every 1-2 days and subcultured when cell reached 80 to $90 \%$ confluence. Cell morphological observation was performed with an ordinary optical microscope (Nikon Corporation, Tokyo, Japan).

Reverse transcription-quantitative polymerase chain reaction $(R T-q P C R)$. Total RNA from tissues or cells were extracted with the TRIzol reagent (Invitrogen; Thermo Fisher Scientific, Inc., Waltham, MA, USA) according to the manufacturer's instructions. For tissue samples, $1 \mathrm{ml}$ TRIzol reagent per $100 \mathrm{mg}$ of tissue sample was used followed by homogenization of tissue samples with a glass Teflon homogenizer (Invitrogen; Thermo Fisher Scientific, Inc.). Then, the homogenized samples were processed to phase separation following the manufacturer's instructions. PCR primers were designed and synthesized by the Shenzhen Huada Gene Science and Technology Services Limited Company, Co., Ltd., (Shenzhen, China). The specific primers used in qRT-PCR were shown in Table I. Relative expression was calculated using the $2^{-\Delta \Delta \mathrm{Cq}}$ method (16).

After transfection with LipofectamineTM2000 plasmid containing over-expressed H19, A549 cells were collected. cDNA synthesis was performed with the one-step method using commercial reverse transcription kit (Fermentas, no. K1633; Wuhan Boster Biological Technology, Ltd., Wuhan, China). qPCR amplification and analysis was performed using ABI 7500 system (Applied Biosystems; Thermo Fisher Scientific, Inc.). The PCR condition was: $95^{\circ} \mathrm{C}$ for $10 \mathrm{~min}, 40$ cycles of $95^{\circ} \mathrm{C}$ for $15 \mathrm{sec}, 60^{\circ} \mathrm{C}$ for $1 \mathrm{~min}$ and finally an elongation step at $72^{\circ} \mathrm{C}$ for $30 \mathrm{sec}$. U6 small RNA and $\beta$-actin was chosen as a loading control for normalization and for quantification of miR-203 and H19 expression.

CCK-8 proliferation assay. A549 cells in each group were collected after transfection and seeded in 96 -well plates at a density of $1 \times 10^{3}$ cells. A final concentration of $10 \%$ of CCK- 8 reagent (Wuhan Biological Co., Ltd., Wuhan, China) was added into cells at 24,48 and $72 \mathrm{~h}$ incubation time. Wavelength value was detected at $450 \mathrm{~nm}$ absorbance using a microplate reader (Bio-Rad Laboratories, Inc., Hercules, CA, USA).

Cell invasion assay. A549 cells in each group were collected after transfection and cultured with serum-free RPMI-1640 medium. Cells was re-suspended and added to the pre-paved Matrigel Transwell chamber (Corning Incorporated, Corning, NY, USA) at a density of $5 \times 10^{4}$ per well. The lower chamber contained $600 \mathrm{ml}$ complete medium. Normal culture was incubated and removed from the upper chamber following $24 \mathrm{~h}$ incubation. Cells ware collected and fixed with $4 \%$ paraformaldehyde. Afterwards, Cells were treated with $0.01 \%$ crystal violet staining to determine the cell count. Cell numbers in the lower chamber were counted from 5 random selected fields at x200 optical microscope. Each experiment was repeated three times.

Western blot analysis. Cells were added with an appropriate amount of RIPA lysis to obtain total cellular protein solution for protein extraction. After treatment with an appropriate amount of SDS buffer at $100^{\circ} \mathrm{C}$ water bath solution, protein samples underwent polyacrylamide gel electrophoresis and transferred to PVDF membranes followed by incubation of primary antibody and secondary antibody (Abcam, Cambridge, UK). Afterwards, the results were analyzed using an enhanced chemiluminescence kit (Cell Signaling Technology, Inc., Danvers, MA, USA) according to the manufacturer's protocols. GAPDH was used for normalization and quantification of protein. Quantification of bands was analyzed using the ImageJ software (National Institutes of Health, Bethesda, MD, USA).

Cell transfection. NSCLC cells were seeded into 6-well plates for $24 \mathrm{~h}$ and grew to $70 \%$ confluence for transfection. The LipofecamineTM2000 mixed with H19 mimic, inhibition or negative control and miR-203 mimic, inhibition or negative control (final concentration of $100 \mathrm{nmol} / \mathrm{l}$ ) were added into cells after cells were incubated for $6 \mathrm{~h}$ and cultured in RPMI-1640 for 24 h. Afterwards, cells were collected for subsequent experiments.

Luciferase reporter gene assay. A density of $1 \times 10^{5}$ cells were seeded in 24-well plates until they reached $70 \%$ confluence. Cells were co-transfected with the LipofectamineTM2000 plasmid which contained the luciferase promoter H19 with overexpressed plasmids. Cells that were cultured for $48 \mathrm{~h}$ were measured and analyzed in accordance with the dual luciferase reporter gene assay kit (Promega Corporation, Madison, WI, USA).

Online prediction of potential target. The miRanda (http://www.microRNA.org) (17), PicTar (https://pictar 
Table I. Quantitative PCR primer sequences.

Forward primer sequence

Reverse primer sequence

\begin{tabular}{lll}
\hline H19 & 5'-GCCTTGACGTGCTGGATCT-3' & 5'-TCCGATGCTTTACTCAAGAAGTT-3' \\
Internal control U6 & 5'-GACGGACACCCTCACTACTG-3' & 5'-GACGTTCATGATTCAAGCATGC-3' \\
miR-203 & 5'-TGCTCTAGAGGCGTCTAAGGCGTCCG-3' & 5'-CCCAAGCTTCACCTCCCAGCAGCACTTG-3'
\end{tabular}
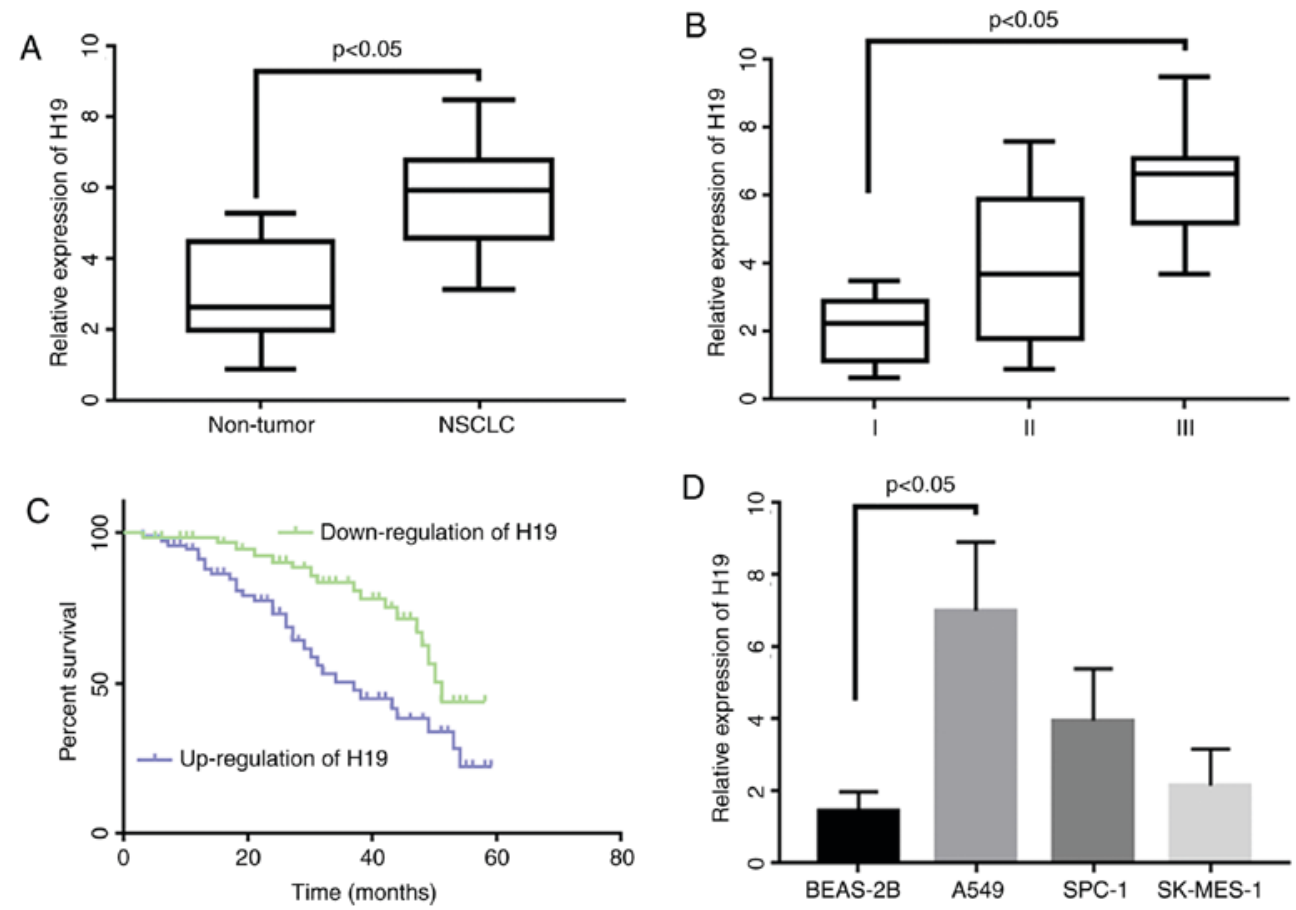

Figure 1. Association of H19 expression and clinicopathological features. (A) H19 expression in 76 NSCLC tissues and adjacent normal tissues. P $<0.05$ compared to normal tissues. (B) Correlation of clinical stages in NSCLC patients and H19 expression. P $<0.05$ compared to stage I. (C) Overall survival analysis according to $\mathrm{H} 19$ expression level. (D) Innate expression level of H19 in different types of lung cancer. $\mathrm{P}<0.05$ compared to normal human bronchial epithelial cells. All experiments were repeated at least twice. NSCLC, non-small cell lung cancer.

.mdc-berlin.de/) (18), TargetScan (http://www.targetscan .org) (19) online prediction tools were used to predict miRNA target genes separately based on previous reports (20-22). Generally, the default settings were adopted according to instructions unless otherwise specified. Taking miRanda for an example, the homo sapiens parameter was selected. Other important parameters were set to individual values, e.g. -8.0 for Gap Open Penalty, -2.0 for Gap Extend, 50.0 for Score Threshold, $-20.0 \mathrm{kcal} / \mathrm{mol}$ for Energy Threshold, and 2.0 for Scaling Parameter. For all three software the average minimum free energy change was measured and used as the reference. Target selection were based on the following criteria: i) Potential target sites were determined by at least two different approaches; ii) target sites must be located in accessible regions and iii) multiple target sites were prioritized (23).

Statistical analysis. Statistical analysis was performed using SPSS v.12.0 statistical software (SPSS Inc., Chicago, IL, USA). Experimental data was presented as Mean \pm SEM (stand error of mean) or in percentages (\%). Comparison between different experimental groups was performed with the two-tailed student's T test and multiple group comparisons used one-way
ANOVA followed by Tukey's post hoc test. Log-rank regression was used to analyze the overall survival curve. $\mathrm{P}<0.05$ was considered to indicate a statistically significant difference.

\section{Results}

Overexpression of $\mathrm{H} 19$ in NSCLC tissues and cells. The expression of H19 was significantly increased in lung cancer tissues compared to adjacent tissues (Fig. 1A). Based on the relative expression level of H19, patients were divided into a high expression (tumor/control $\geq 1.5$ ) and a low expression group (tumor/control <1.5). It was found that patients in the higher expression group presented with advanced clinical stages in comparison to the lower expression group (Table II and Fig. 1B). The overall survival time of patients in the higher expression group was also shorter compared to the lower expression group (Fig. 1C). In addition, the study examined the H19 expression levels in different NSCLC cells. The results showed that H19 in A549, SPC-A1 and SK-MES-1 cells increased by 4.2-fold, 3.4-fold and 2.6-fold in contrast to the normal bronchial epithetical cell line, BEAS (Fig. 1D). 
Table II. Association of clinicalpathological features and H19 expression in NSCLC patients.

\begin{tabular}{|c|c|c|c|c|c|c|}
\hline \multirow[b]{2}{*}{ Characteristics } & \multirow[b]{2}{*}{$\mathrm{N}=76$} & \multicolumn{4}{|c|}{ H19 expression } & \multirow[b]{2}{*}{ P-value } \\
\hline & & Low & $\%$ & High & $\%$ & \\
\hline Age (years) & & 41 & & 35 & & 0.692 \\
\hline$<60$ & 28 & 15 & 36.59 & 13 & 37.14 & \\
\hline$\geq 60$ & 48 & 26 & 63.41 & 22 & 62.86 & \\
\hline Sex & & & & & & 0.577 \\
\hline Male & 32 & 17 & 41.46 & 15 & 42.86 & \\
\hline Female & 44 & 24 & 58.54 & 20 & 57.14 & \\
\hline Smoking & & & & & & 0.107 \\
\hline Yes & 21 & 11 & 26.83 & 10 & 28.57 & \\
\hline No & 55 & 30 & 73.17 & 25 & 71.43 & \\
\hline TNM & & & & & & 0.024 \\
\hline I & 42 & 26 & 63.41 & 16 & 45.71 & \\
\hline II & 24 & 11 & 26.83 & 13 & 31.71 & \\
\hline III & 10 & 4 & 9.76 & 6 & 22.58 & \\
\hline Tumor size $(\mathrm{cm})$ & & & & & & 0.085 \\
\hline$<5$ & 49 & 25 & 60.98 & 24 & 68.57 & \\
\hline$\geq 5$ & 27 & 16 & 39.02 & 11 & 31.43 & \\
\hline Pathological histology & & & & & & 0.142 \\
\hline Squamous cell carcinoma & 47 & 26 & 63.41 & 21 & 60 & \\
\hline Adenocarcinoma & 19 & 8 & 19.51 & 11 & 26.83 & \\
\hline Large cell carcinoma & 10 & 7 & 17.07 & 3 & 13.17 & \\
\hline \multicolumn{7}{|l|}{ Survival time (months) } \\
\hline$<24$ & 45 & 20 & 48.78 & 25 & 71.43 & 0.021 \\
\hline$\geq 24$ & 31 & 21 & 51.22 & 10 & 28.57 & \\
\hline
\end{tabular}

NSCLC, non-small cell lung cancer.

Overexpression of $H 19$ can promote proliferation and EMT process in A549. Based on the expression results, A549 cell line was chosen for another functional study. Cells were transfected with $\mathrm{H} 19$ plasmid and validated by quantitative real time polymerase chain reaction (qRT-PCR). The results showed that the expression level of H19 in transfected cells increased more than 20-fold than control cells $(\mathrm{P}<0.05$; Fig. 2A). Cell proliferation results indicated that cell proliferation became significantly enhanced in H19 over expressed cells in contrast to the control group following $72 \mathrm{~h}$ incubation period (Fig. 2B). The invasion results demonstrated that the number of crossing members were significantly increased in H19 overexpression group when compared to negative control group $(\mathrm{P}<0.05$; Fig. $2 \mathrm{C}$ and $\mathrm{D})$.

Association of miR-203 with H19 and EMT expression. EMT is a well-documented event in the progression of tumor invasion. Afterwards, western blot assays were used to detect the expression of EMT markers (CDH1, N-cadherin and Vimentin, SNAI1, SNAI2, and ZEB1) between H19 overexpressed cells and control cells. Western blot testing found that epithelial marker CDH1 was significantly reduced after H19 overexpression. In contrast, the expressions of mesenchymal markers which included SNAI1 and Vimentin, were increased (Fig. 3A).
Accumulative studies have revealed that IncRNA H19 mediate EMT by functioning as a sponge for the miRNAs in a variety of cancers (24-27). Recently, miR-203 was reported to play a crucial role in the EMT core network, which functions as a switch controlling epithelial cell plasticity during cancer progression (28). We hypothesized that H19 could possibly promote EMT by acting as a miRNA sponge and hijack miR-203 since miR-203 is known to attenuate EMT in many types of cancers. In order to search for potential targets of miR-203, three online prediction tools were used such as, miRanda, PicTar and TargetScan, and we identified putative miR-203 binding site in 3'-UTR of H19 as a potential target. We also referred to the previously predicted miRNA targets for H19 by others, e.g. RGD (https://rgd.mcw.edu/) (29) which results are in agreement with our prediction.

Afterwards, a luciferase reporter was constructed containing wild type and mutant H19 3'-UTR binding sequence (Fig. 3B). After transfection, miR-203 overexpressed plasmid and $\mathrm{H} 19$ gene promoter were detected by luciferase activity and the results are shown in Fig. 3B. Additionally, the luciferase activity was significantly inhibited, as its expression decreased approximately by $60 \%$. miR-203 inhibitor significantly increased the luciferase activity of H19 in compared with the control cells $(\mathrm{P}<0.05)$. Furthermore, we observed that miR-203 

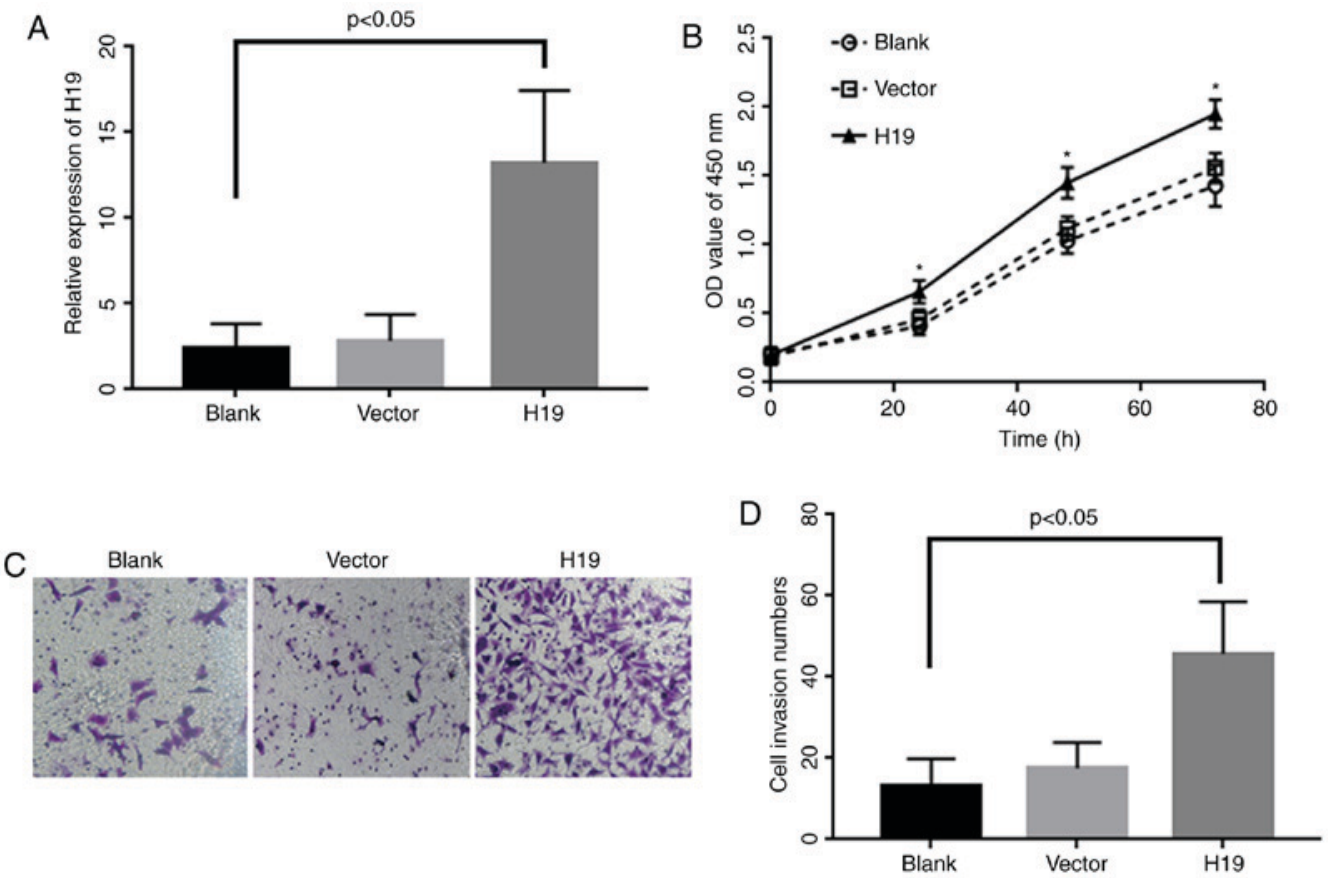

Figure 2. The effect of over expressed H19 on cell function. (A) Validation of over expression of H19 efficiency with qRT-PCR. Three independent experiments performed in triplicate. $\mathrm{P}<0.05$ compared to the blank control. (B) Ectopic expression of $\mathrm{H} 19$ enhanced cell proliferation. The data was performed in triplicate wells for each time point and repeated at least twice. "P $<0.05$ compared to the blank control. (C and D) The effect of H19 over expression on cell invasion ability (magnification, $\mathrm{x} 200$ ). Three independent experiments performed in triplicate. $\mathrm{P}<0.05$ compared to the blank control.

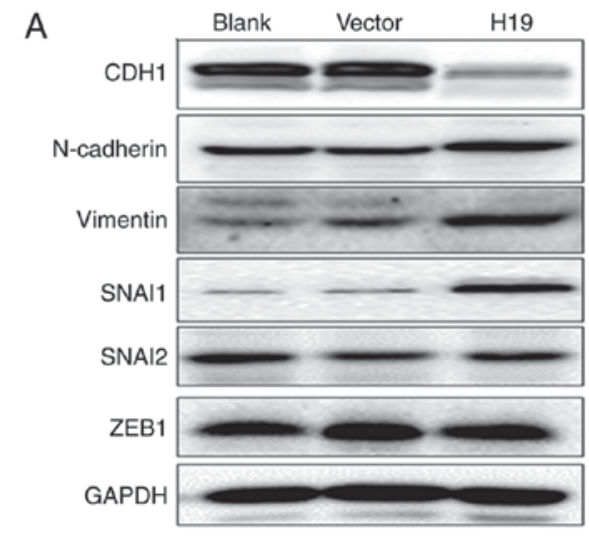

B miR-203: 3' -GGUAGAAAUGGUCUGUCACAAAU- 5' H19-Wt: $5^{\prime}$-AGAAUUCAUACCAAUCAGUGUUG- $3^{\prime}$ H19- Mut: 5' -AGAAUUCAUACCAAUCUGAGAUG- $3^{\prime}$
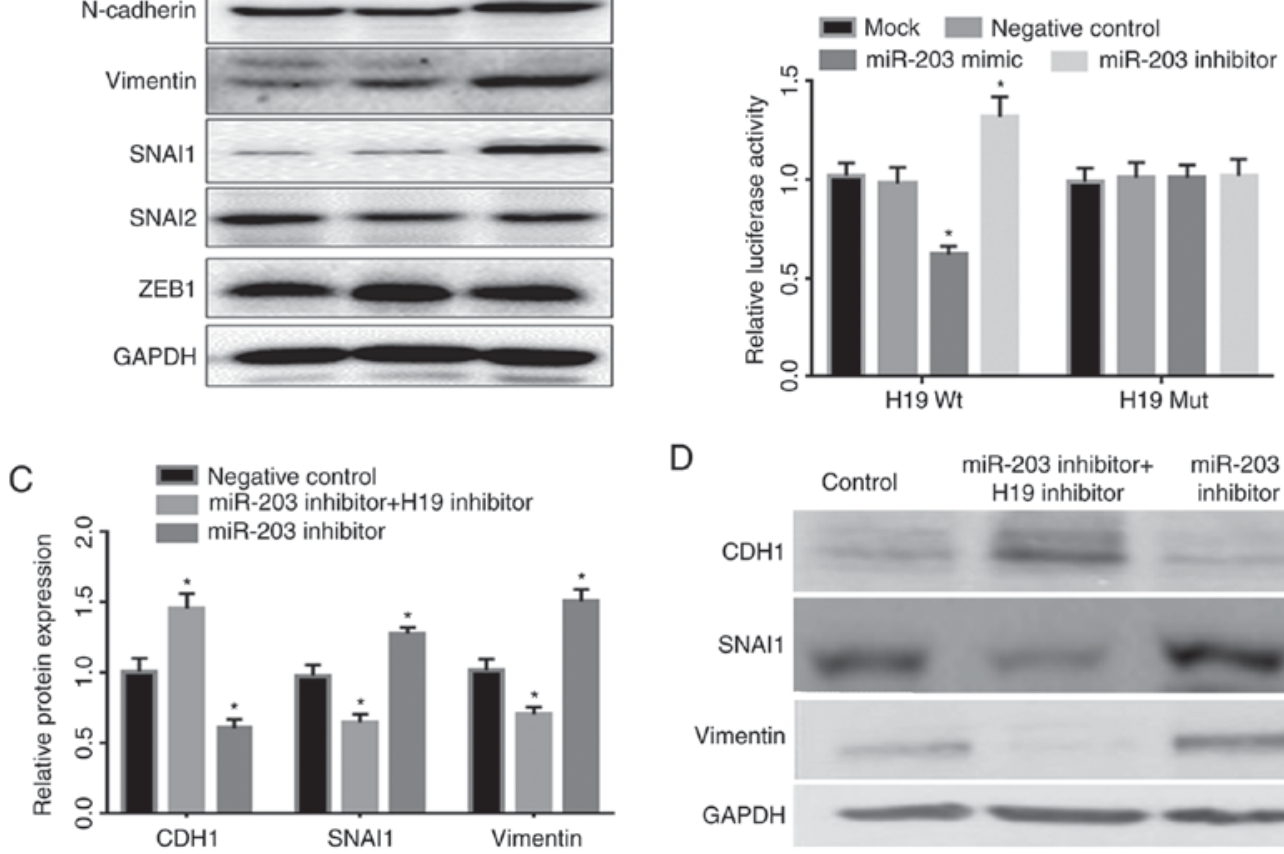

D

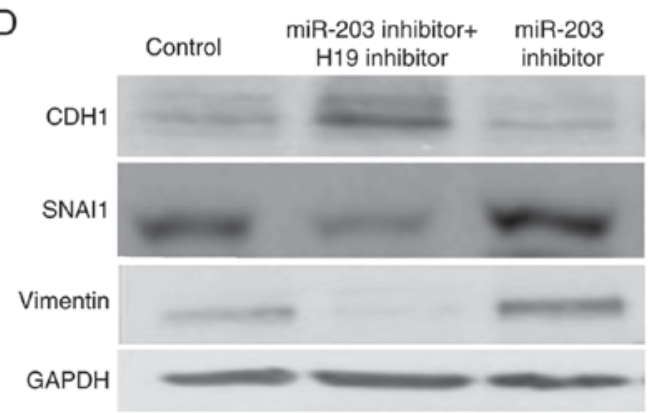

Figure 3. Involvement of H19 in miR-203 induced EMT process. (A) Alteration of EMT marker proteins in H19 over expressed cells. (B) Luciferase assay was performed to validate the direct binding effect of miR-203 on H19. The data was representative of at least three independent experiments. "P<0.05 compared to the negative control. (C) Down-regulation of H19 attenuated miR-203 inhibitor induced EMT process. The data was representative of at least three independent experiments. " $\mathrm{P}<0.05$ compared to the negative control. (D) Alteration of EMT marker proteins in A549 cells following miR-203 inhibitor and/or H19 inhibitor transfection. EMT, epithelial-mesenchymal transition.

inhibitor suppressed the expression of epithelial marker CDH1 and promoted the expression of mesenchymal markers, SNAI1 and Vimentin. However, co-transfection of miR-203 inhibitor and H19 inhibitor reversed the effect (Fig. 3C and D). 

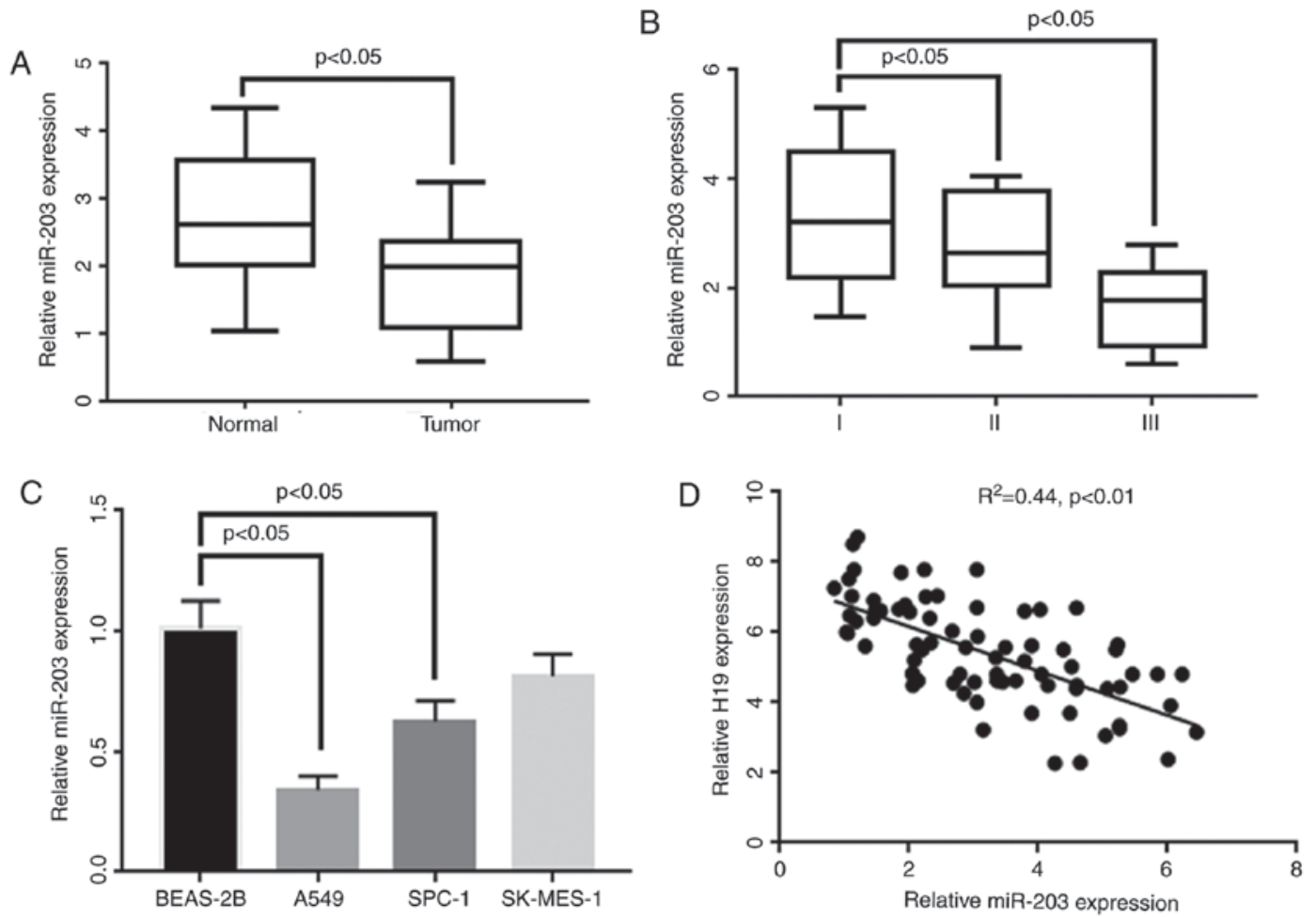

Figure 4. Determination of miR-203 expression in NSCLC tissues and cell lines. (A) Decreased expression of miR-203 was observed in NSCLC tissue samples compared with paired normal tissue samples. Experiments were run in triplicates. $\mathrm{P}<0.05$ compared to normal tissue. (B) A negative association of miR-203 expression level and TNM stages. Experiments were run in triplicates. $\mathrm{P}<0.05$ compared to stage I. (C) miR-203 expression levels in three NSCLC cell lines (A549, SPC-A1, SKMES-1) and a normal bronchial epithelial cell line BEAS-2B. miR-203 was significantly down-regulated in all cell lines compared to BEAS-2B. P<0.05 compared to normal cells. The experiments were carried out in triplicate. (D) Association between the expressions of miR-203 and H19 in NSCLC tissue samples, $\mathrm{r}^{2}=0.44, \mathrm{P}<0.01$. NSCLC, non-small cell lung cancer.
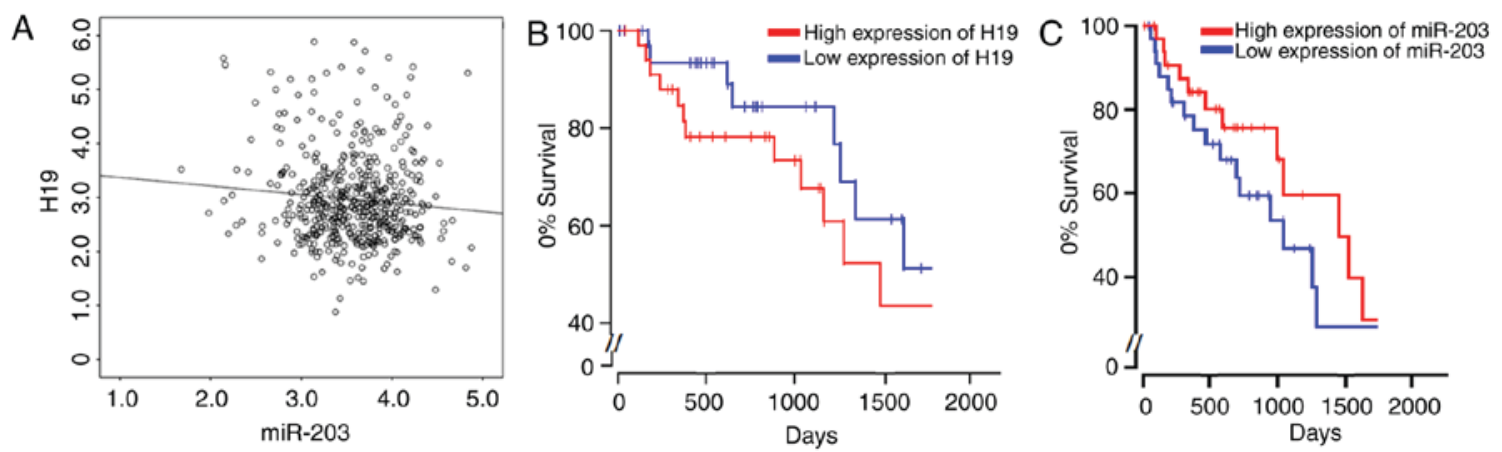

Figure 5. Expression and survival analysis with public TCGA dataset. (A) Negative correlation between the expressions of H19 and miR-203 in LUAD. TCGA database was employed to retrieve the lncRNA and miRNA expression profiles. Scattering plot shows the distribution of H19 vs. miR-203 expression for each sample with LUAD in TCGA database. (B) Kaplan-Meier plot for both high and low expressions of H19 in LUAD. (C) Kaplan-Meier plot for both high and low expressions of miR-203. The upper and lower percentiles were set to seven for the comparison of Kaplan-Meier plots between different expressions of target genes. LUAD, lung adenocarcinoma; TCGA, The Cancer Genome Atlas; lncRNA, long non-coding RNA.

Down-regulation of miR-203 in tissue samples and NSCLC cell lines. As shown in Fig. 4A, a decreased expression of miR-203 was observed in NSCLC tissue samples compared with paired normal tissue samples (Fig. 4A). Further analysis revealed a negative association of miR-203 expression level and TNM stage (Fig. 4B). Next, we determined the miR-203 expression levels in three NSCLC cell lines (A549, SPC-A1, SKMES-1) and in the normal bronchial epithelial cell line BEAS-2B. Our results found that miR-203 was significantly down-regulated in all cell lines compared to BEAS-2B (Fig. 4C). Based on the observed negative relation between H19 and miR-203, our results suggested an association of miR-203 expression with H19 expression in NSCLC samples (Fig. 4D).

Given the limited number of samples, we also turned to the public dataset of TCGA (https://cancergenome.nih.gov/) and studied the expression profiles of both H19 and miR-203 in lung adenocarcinoma (LUAD), one largest subtype of NSCLC which accounts for $40 \%$ of all total lung cancer cases (30). Our results from analysis are consistent with the above findings, indicating a negative correlation between the expressions of $\mathrm{H} 19$ and miR-203, which was proved with statistical significance $(\mathrm{P}<0.05$; Fig. 5A). Moreover, 
we also determined the Kaplan-Meier plots for both high and low expressions of $\mathrm{H} 19$ and miR-203. It was shown that high expression of $\mathrm{H} 19$ results in the significant reduction of cumulative survival among LUAD patients (Fig. 5B), while high expression of miR-203 largely improves the cumulative survival (Fig. 5C). All these results provide a convincing support for the current work.

\section{Discussion}

With the completion of the Human Genome Project, it was discovered that in addition to approximately 20,000 protein-coding genes that account for $2 \%$ of the whole genome, there are a large number of non-coding RNAs (ncRNAs) containing microRNA and lncRNA.16 Also, ncRNAs are reported to participate in a variety of functional cell processes on multiple levels including protein translation and protein degradation. Despite the evidence supporting the function of ncRNA in carcinogenesis, their role and potential application in lung cancer is still in its early stages (31-34).

As a transcriptional regulatory lncRNA, H19 was originally found in embryonic development related research (12). In recent years, a series of studies have found that H19 abnormally over-expressed in many tumors, such as bladder cancer (35), gastric cancer (36), hepatocellular carcinoma (37), and NSCLC $(38,39)$. Also, an in-vitro study revealed that H19 overexpression promotes the proliferation of tumor, increases migration and invasion of tumor cells and reduces sensitivity to chemotherapy, thus suggesting an important role of H19 in tumorigenesis and cancer development (40). However, the expression of $\mathrm{H} 19$ and its function in NSCLC is not yet clear.

The results of our study indicated that compared with normal tissues, H19 was significantly up regulated in NSCLC tissues, suggesting its oncogenic role in NSCLC cancer occurrence and development. Further analysis demonstrated that H19 over-expression is closely associated with an advanced clinical stage and an aggressive lymph node metastasis in patients, suggesting that a high expression of H19 may be involved in malignant lung cancer proliferation. Overall survival analysis found that high expression is related to poor prognosis in patients, suggesting a potential application of H19 as a prognostic biomarker in NSCLC.

Our results showed that overexpression of H19 increased cell numbers of crossing basement members which suggested an enhanced cell invasiveness ability. The present study also found that A549 cells following an overexpression of H19 demonstrated significant morphological changes with a shuttle-shaped oval deformation growth, increased pseudopodia variable length and a larger cell gap. All these changes indicated involvement of the EMT process. It is well known that EMT is a complex and an important biological process that participates in a variety of pathophysiological processes including cancer invasion and transformation $(41,42)$. When tumor cells undergo EMT, they lose their polarity and increase migration and invasion ability. Therefore, we analyzed the changes of EMT related proteins to confirm whether EMT stimulates the effect of $\mathrm{H} 19$ on cell invasion. The results showed a decreased expression of epithelial marker CDH1 and increased expression of mesenchymal markers, SNAI1 and Vimentin along with an over expression of H19 suggesting a mediatory role of EMT in enhanced cell invasion.

Previous studies provided evidence of a decreased expression of miR-203 in a variety of lung cancer tissue samples in contrast to the expression of normal human bronchial epithelial cells or normal lung tissues, respectively $(43,44)$. Also, reduced expression of miR-203 was found to be associated with metastatic tumors (45). Additionally, over-expression of miR-203 in various cancer cell lines led to inhibition of EMT processes, such as cell proliferation, migration, invasion and tumor metastasis (46). Furthermore, previous studies demonstrated the association of $\mathrm{H} 19$ with miRNA ribonucleoprotein complexes, thus acting as a natural molecular sponge for various miRNAs (47-49). Also, studies showed lncRNAs have the ability to modulate downstream targets of miRNA by acting as a decoy to sequester miRNAs (50-52). Given that EMT is attenuated by miR-203 in many cancer types, we hypothesized that $\mathrm{H} 19$ acts as a miRNA sponge and hijacks miR-203 hence promotes EMT in the process.

Finding of our study revealed that H19 expression was elevated in NSCLC tissues along with a decreased miR-203 expression level. It was also found that patients in advanced clinical stages had a higher H19 and a lower miR-203 expression compared normal tissues. The overall survival time of patients in higher H19 expression group was shorter in contrast to the lower H19 expression group. Up-regulation of H19 enhanced cell proliferation and promoted invasion. Over expression of H19 stimulated the EMT process in lung cancer cells and presented with typical morphological characteristics of EMT. The level of mesenchymal marker proteins such as Vimentin and SNAI1 increased; while CDH1 protein level decreased. Also, H19 negatively regulated miR-203. Inhibition of H19 attenuated miR-203 induced EMT process. Our study also reported a negative correlation between miR-203 and H19 expression level in tissue samples and cell lines. In addition, a decreased miR-203 expression was associated with the TNM stage. Furthermore, inhibition of H19 significantly reversed the EMT promotion effect of miR-203 on lung cancer cells through down-regulation of epithelial markers and an up-regulation of mesenchymal markers. In short, H19 may promote invasion in NSCLC by influencing the EMT process, which can affect regulation of specific miRNAs.

In summary, $\mathrm{H} 19$ overexpression can induce the occurrence of EMT and promote invasion in lung cancer cells. The direct binding effect of miR-203 and H19 on EMT process suggested that miRNA-IncRNA acts as a potential regulatory network in H19-mediated EMT changes. Therefore, our study provides evidence that $\mathrm{H} 19$, which is involved in the tumorigenesis of NSCLC, can serve as a potential target for new drugs and clinical biomarkers.

\section{Acknowledgements}

Not applicable.

\section{Funding}

The present study was supported by National Nature Science Foundation of China (grant no. 81560487). 


\section{Availability of data and materials}

The data used and analyzed during the current study are available from the corresponding author on reasonable request.

\section{Authors' contributions}

XJG contributed to the present study design and performed the majority of the experiments. LMZ and ZXF contributed to the data analysis and data interpretation. LL, YJZ, MYL and JYJ contributed to collection of the clinical samples.

\section{Ethics approval and consent to participate}

The present study was approved by the Ethics Committee of Zunyi Medical University. Informed consent was obtained from each patient prior to surgery. All experimental procedures were carried out in accordance with the approved guidelines and were in agreement with the Declaration of Helsinki.

\section{Patient consent for publication}

Not applicable.

\section{Competing interests}

The authors declare that they have no competing interests.

\section{References}

1. Siegel RL, Miller KD and Jemal A: Cancer statistics, 2016. CA Cancer J Clin 66: 7-30, 2016.

2. Rosell R and Karachaliou N: Lung cancer in 2014: Optimizing lung cancer treatment approaches. Nat Rev Clin Oncol 12: 75-76, 2015.

3. Pérez-Soler R: Individualized therapy in non-small-cell lung cancer: Future versus current clinical practice. Oncogene 28 (Suppl 1): S38-S45, 2009.

4. Lin S and Gregory RI: MicroRNA biogenesis pathways in cancer. Nat Rev Cancer 15: 321-333, 2015.

5. Cai Y, Yu X, Hu S and Yu J: A brief review on the mechanisms of miRNA regulation. Genomics Proteomics Bioinformatics 7: 147-154, 2009.

6. Ha M and Kim VN: Regulation of microRNA biogenesis. Nat Rev Mol Cell Biol 15: 509-524, 2014.

7. Jansson MD and Lund AH: MicroRNA and cancer. Mol Oncol 6: 590-610, 2012.

8. Reddy KB: MicroRNA (miRNA) in cancer. Cancer Cell Int 15: 38,2015

9. Bartel DP: MicroRNAs: Target recognition and regulatory functions. Cell 136: 215-233, 2009.

10. Kong YW, Ferland-McCollough D, Jackson TJ and Bushell M: microRNA in cancer management. Lancet Oncol 13: e249-e258, 2012.

11. Slack FJ and Weidhaas JB: MicroRNA in cancer prognosis N Engl J Med 359: 2720-2722, 2008.

12. Brannan CI, Dees EC, Ingram RS and Tilghman SM: The product of the H19 gene may function as an RNA. Mol Cell Biol 10: 28-36, 1990.

13. Vennin C, Spruyt N, Dahmani F, Julien S, Bertucci F, Finetti P, Chassat T, Bourette RP, Le Bourhis X and Adriaenssens E: H19 non coding RNA-derived miR-675 enhances tumorigenesis and metastasis of breast cancer cells by downregulating c-Cbl and Cbl-b. Oncotarget 6: 29209-29223, 2015.

14. Zhou X, Yin C, Dang Y, Ye F and Zhang G: Identification of the long non-coding RNA H19 in plasma as a novel biomarker for diagnosis of gastric cancer. Sci Rep 5: 11516, 2015.
15. Matouk IJ, Halle D, Gilon M and Hochberg A: The non-coding RNAs of the H19-IGF2 imprinted loci: A focus on biological roles and therapeutic potential in Lung Cancer. J Transl Med 13: 113,2015

16. Livak KJ and Schmittgen TD: Analysis of relative gene expression data using real-time quantitative PCR and the 2(-Delta Delta C(T)) method. Methods 25: 402-408, 2001.

17. John B, Enright AJ, Aravin A, Tuschl T, Sander C and Marks DS: Human MicroRNA targets. PLoS Biol 2: e363, 2004.

18. Krek A, Grün D, Poy MN, Wolf R, Rosenberg L, Epstein EJ, MacMenamin P, da Piedade I, Gunsalus KC, Stoffel M and Rajewsky N: Combinatorial microRNA target predictions. Nat Genet 37: 495-500, 2005.

19. Lewis BP, Shih IH, Jones-Rhoades MW, Bartel DP and Burge CB: Prediction of mammalian microRNA targets. Cell 115: 787-798, 2003.

20. Hariharan M, Scaria V, Pillai B and Brahmachari SK: Targets for human encoded microRNAs in HIV genes. Biochem Biophys Res Commun 337: 1214-1218, 2005.

21. Grün D, Wang YL, Langenberger D, Gunsalus KC and Rajewsky N: microRNA target predictions across seven Drosophila species and comparison to mammalian targets. PLoS Comput Biol 1: e13, 2005.

22. Reid JG, Nagaraja AK, Lynn FC, Drabek RB, Muzny DM, Shaw CA, Weiss MK, Naghavi AO, Khan M, Zhu H, et al: Mouse let-7 miRNA populations exhibit RNA editing that is constrained in the 5'-seed/cleavage/anchor regions and stabilize predicted mmu-let-7a:mRNA duplexes. Genome Res 18: 1571-1581, 2008.

23. Chan WL, Huang HD and Chang JG: IncRNAMap: A map of putative regulatory functions in the long non-coding transcriptome. Comput Biol Chem 50: 41-49, 2014.

24. Zhou W, Ye XL, Xu J, Cao MG, Fang ZY, Li LY, Guan GH, Liu Q, Qian YH and Xie D: The lncRNA H19 mediates breast cancer cell plasticity during EMT and MET plasticity by differentially sponging miR-200b/c and let-7b. Sci Signal 10: pii: eaak9557, 2017.

25. Zhang Q, Li X, Li X, Li X and Chen Z: LncRNA H19 promotes epithelial-mesenchymal transition (EMT) by targeting miR-484 in human lung cancer cells. J Cell Biochem 119: 4447-4457, 2018.

26. Liang WC, Fu WM, Wong CW, Wang Y, Wang WM, Hu GX, Zhang L, Xiao LJ, Wan DC, Zhang JF and Waye MM: The lncRNA H19 promotes epithelial to mesenchymal transition by functioning as miRNA sponges in colorectal cancer. Oncotarget 6: 22513-22525, 2015.

27. Lv M, Zhong Z, Huang M, Tian Q, Jiang R and Chen J: IncRNA H19 regulates epithelial-mesenchymal transition and metastasis of bladder cancer by miR-29b-3p as competing endogenous RNA. Biochim Biophys Acta 1864: 1887-1899, 2017.

28. Moes M, Le Béchec A, Crespo I, Laurini C, Halavatyi A, Vetter G, Del Sol A and Friederich E: A novel network integrating a miRNA-203/SNAI1 feedback loop which regulates epithelial to mesenchymal transition. PLoS One 7: e35440, 2012.

29. Shimoyama M, De Pons J, Hayman GT, Laulederkind SJ, Liu W, Nigam R, Petri V, Smith JR, Tutaj M, Wang SJ, et al: The Rat Genome Database 2015: Genomic, phenotypic and environmental variations and disease. Nucleic Acids Res 43 (Database Issue): D743-D750, 2015.

30. Chang JT, Lee YM and Huang RS: The impact of the Cancer Genome Atlas on lung cancer. Transl Res 166: 568-585, 2015.

31. Fatima R, Akhade VS, Pal D and Rao SM: Long noncoding RNAs in development and cancer: Potential biomarkers and therapeutic targets. Mol Cell Ther 3: 5, 2015.

32. Gao Y, Gao F, Ma JL, Sun WZ and Song LP: The potential clinical applications and prospects of microRNAs in lung cancer. Onco Targets Ther 7: 901-906, 2014.

33. Ricciuti B, Mecca C, Crinò L, Baglivo S, Cenci M and Metro G: Non-coding RNAs in lung cancer. Oncoscience 1: 674-705, 2014.

34. Sana J, Faltejskova P, Svoboda M and Slaby O: Novel classes of non-coding RNAs and cancer. J Transl Med 10: 103, 2012.

35. Ariel I, Sughayer M, Fellig Y, Pizov G, Ayesh S, Podeh D, Libdeh BA, Levy C, Birman T, Tykocinski ML, et al: The imprinted H19 gene is a marker of early recurrence in human bladder carcinoma. Mol Pathol 53: 320-323, 2000.

36. Li H, Yu B, Li J, Su L, Yan M, Zhu Z and Liu B: Overexpression of IncRNA H19 enhances carcinogenesis and metastasis of gastric cancer. Oncotarget 5: 2318-2329, 2014.

37. Zhang L, Yang F, Yuan JH, Yuan SX, Zhou WP, Huo XS, Xu D, Bi HS, Wang F and Sun SH: Epigenetic activation of the MiR-200 family contributes to H19-mediated metastasis suppression in hepatocellular carcinoma. Carcinogenesis 34: 577-586, 2013. 
38. Zhang E, Li W, Yin D, De W, Zhu L, Sun S and Han L: c-Myc-regulated long non-coding RNA H19 indicates a poor prognosis and affects cell proliferation in non-small-cell lung cancer. Tumour Biol 37: 4007-4015, 2016.

39. Cui JD, Mo J, Luo M, Yu Q, Zhou S, Li T, Zhang Y and Luo W: c-Myc-activated long non-coding RNA H19 downregulates miR-107 and promotes cell cycle progression of non-small cell lung cancer. Int J Clin Exp Pathol 8: 12400-12409, 2015.

40. Matouk IJ, DeGroot N, Mezan S, Ayesh S, Abu-lail R, Hochberg A and Galun E: The H19 non-coding RNA is essential for human tumor growth. PLoS One 2: e845, 2007.

41. Lin CW, Lin PY and Yang PC: Noncoding RNAs in tumor epithelial-to-mesenchymal transition. Stem Cells Int 2016: 2732705, 2016

42. Heerboth S, Housman G, Leary M, Longacre M, Byler S, Lapinska K, Willbanks A and Sarkar S: EMT and tumor metastasis. Clin Transl Med 4: 6, 2015.

43. Jin J, Deng J, Wang F, Xia X, Qiu T, Lu W, Li X, Zhang H, Gu X, Liu Y, et al: The expression and function of microRNA-203 in lung cancer. Tumour Biol 34: 349-357, 2013.

44. Tang R, Zhong T, Dang Y,Zhang X, Li P and Chen G: Association between downexpression of MiR-203 and poor prognosis in non-small cell lung cancer patients. Clin Transl Oncol 18: 360-368, 2016

45. Funamizu N, Lacy CR, Kamada M, Yanaga K and Manome Y: MicroRNA-203 induces apoptosis by upregulating Puma expression in colon and lung cancer cells. Int J Oncol 47: 1981-1986, 2015.

46. Ding X, Park SI, McCauley LK and Wang CY: Signaling between transforming growth factor $\beta$ (TGF- $\beta$ ) and transcription factor SNAI2 represses expression of microRNA miR-203 to promote epithelial-mesenchymal transition and tumor metastasis. J Biol Chem 288: 10241-10253, 2013.
47. Zhou X, Ye F, Yin C, Zhuang Y, Yue G and Zhang G: The interaction between MiR-141 and lncRNA-H19 in regulating cell proliferation and migration in gastric cancer. Cell Physiol Biochem 36: 1440-1452, 2015.

48. Zhu M, Chen Q, Liu X, Sun Q, Zhao X, Deng R, Wang Y, Huang J, Xu M, Yan J and Yu J: lncRNA H19/miR-675 axis represses prostate cancer metastasis by targeting TGFBI. FEBS J 281: 3766-3775, 2014.

49. Zhuang M, Gao W, Xu J, Wang P and Shu Y: The long non-coding RNA H19-derived miR-675 modulates human gastric cancer cell proliferation by targeting tumor suppressor RUNX1. Biochem Biophys Res Commun 448: 315-322, 2014.

50. Shi Y, Wang Y, Luan W, Wang P, Tao T, Zhang J, Qian J, Liu N and You Y: Long non-coding RNA H19 promotes glioma cell invasion by deriving miR-675. PLoS One 9: e86295, 2014.

51. Imig J, Brunschweiger A, Brümmer A, Guennewig B, Mittal N, Kishore S, Tsikrika P, Gerber AP, Zavolan M and Hall J: miR-CLIP capture of a miRNA targetome uncovers a lincRNA H19-miR-106a interaction. Nat Chem Biol 11: 107-114, 2015.

52. Cai X and Cullen BR: The imprinted H19 noncoding RNA is a primary microRNA precursor. RNA 13: 313-316, 2007.

This work is licensed under a Creative Commons Attribution-NonCommercial-NoDerivatives 4.0 International (CC BY-NC-ND 4.0) License. 